\title{
Formulation and Evaluation of Valsartan
}

\section{Oral Granule}

\begin{tabular}{|c|c|c|}
\hline Anoosh Bashir Hagop ${ }^{1 *}$ & Anjam Hama Abdalla ${ }^{1}$ & Hewa Abdulla Hamadameen ${ }^{1}$ \\
\hline & Abstract & \\
\hline
\end{tabular}

Background and objective: Oral dosage form is a popular route of drug delivery and the most common types are tablets and capsules because of their ease administration, patient acceptance and stability of formulation, while administration of such dosage forms have drawback such as difficulty in swallowing in pediatric and geriatric patients. Oral granules dosage form as sachet or dry syrup of valsartan is an alternative dosage form to be used.

Methods: Wet granulation method was used to prepare two different formulations of valsartan granules, novel formula (F1) and conventional formula (F2). The two formulas prepared by the same method with different technique of mixing. Where F1 was prepared by dissolving the drug in limited quantity of ethanol and PVP in D.W separately then both solutions were mixed and added to the remaining dry ingredients slowly to form a granules after sieving and drying process in the oven. While in the case of F2 all dry ingredients including the drug were mixed together except the PVP which dissolved separately in D.W then the prepared solution was added slowly and the granules were formed after sieving and drying the prepared mixture in oven.

Results: The study revealed that F1 has accepted value of dissolution profile where within 30 minutes about $96.84 \%$ of drug was released from F1 which is within the acceptable limit, while for F2 only $64.60 \%$ of the drug was released within the same time. This differences in the release profile was statistically significant $(P<0.05)$ between $\mathrm{F} 1$ and $\mathrm{F} 2$, but a non- significant difference $(P>0.05)$ was seen between $\mathrm{F} 2$ and conventional valsartan tablet using diovan as a reference.

Conclusion: The study concluded that formulation of F1 dry granule can be used as an alternative method to prepare dry granules and to be used by pediatrics and geriatrics patients instead of tablets and capsules. While F2 needs improvement in solubility before using in the preparation of poorly water soluble drugs like valsartan which is within class II BCS (low solubility and high permeability).

Keyword: Valsartan; Sachet; Wet granulation method; Oral granule.

\section{Introduction}

About one third of hypertensive patients will reached goal blood pressures of $<140 / 90$ $\mathrm{mm} \mathrm{Hg}$ with monotherapy, regardless of the medication used. $^{1}$ Valsartan is an angiotensin II-receptor antagonist that has been available overseas for several years which can be use to decrease blood pressure by the same ratio such as other angiotensin II-receptor antagonists. This class of antihypertive drug have same blood pressure lowering effects to those of other classes of antihypertensive drug. ${ }^{2}$ Valsartan is effective in treatment of pediatric, adolescents and the elderly patients with mild to moderate hypertension. ${ }^{3}$ There are several classes of pharmaceutical dosage forms, an oral route is the most accepted route of administration. Tablets and capsules are unsuitable for given large doses of medicine, since single high dose is difficult to swallow, or needed the administration of several pills or capsules at the sometime,

${ }^{1}$ Department of Pharmaceutics, College of Pharmacy, Hawler Medical University, Erbil, Iraq.

* Correspondence: Anush.Hakub@hmu.edu.krd 
it is not accepted by patients. The chewing process need teeth, taste cannot be masked completely and lack of control release probability chewable tablets are not ideal with pediatric and geriatric patients. Hence it is essential to develop a reconstitutable granule dosage form. ${ }^{4}$ Oral granules are solid dosage form that can be reconstituted by the addition of water to administer by oral route. Mostly antibiotics, some moisture sensitive and pediatric drugs are available in the form of dry granules and prepared as dry powder mixtures or granules that are intended to be suspended in water or some other vehicle before oral administration. Oral granules can offer many positive points like remaining of the chemical stability of the active compounds until reconstitution at the time of use and can be easily given to children of different ages by adjusting the volume to swallow. ${ }^{5,6}$ Hence, the aim of this study is to prepare a new dosage form of valsartan as oral granules and sachet by wet granulation method to be used as alternative dosage form that can occupy the place of the conventional valsartan tablets and also to study different parameters affecting dissolution behaviour of the prepared formulas (F1 and F2) then comparing it with that of valsartan conventional tablet (Diovan ${ }^{\circledR} 80 \mathrm{mg}$ tablet) as a reference tablet.

\subsection{Advantages of oral granules for oral use $^{6,7}$}

- Accurate single dosing as the dose is packed in single dose sachets making the formulation easy to carry and enhanced convenience of single dosage regimen.

- Drug dose is relatively independent of any physical factors like temperature, sedimentation rate and liquid flow properties.

- Colored, flavored, sweetened formulation is advantageous for administration to the pediatric population.

- Stable on storage and when reconstituted with an ingestible liquid for administration.

\subsection{Required characteristics of oral} granules for reconstitution ${ }^{8,9}$

- Powder blend must be a uniform mixture of the suitable concentration of each ingredient and during reconstitution.

-The powder blend must disperse rapidly and completely in the aqueous vehicle.

- Final product must have an acceptable appearance, odor and taste.

\subsection{Rapidly dispersed excipients on} reconstitution ${ }^{9}$

- Granule disintegrant: It results in prevention of the particles aggregation.

- Granule binder: It helps to reduce the settling of particles in suspensions and also used as a stabilizer such as high molecular weight povidone.

- Suspending agents: It should be easily dispersed during reconstitution. Some of the suspending agents that are recommended for use are acacia, carboxymethylcellulose sodium and xanthan gum.

- Sweeteners: Sweeteners can mask the unfavorable taste and enhance patient acceptance in the pediatric population that uses this product. It is a significant component of granules for reconstitution such as sucrose, mannitol, dextrose and aspartame.

- Wetting agents: Many drugs are hydrophobic; they repel water and are not easily wetted. The appropriate wetting agent must be selected for optimum dispersion of the drug at the lowest effective concentrations such as polysorbate 80 and sodium lauryl sulfate.

- Other excipients: The other excipients include buffers, preservatives, flavors and colors. ${ }^{10}$

1.4. Method of preparation of dry mixture ${ }^{11}$

- Direct mixing method: it is the easiest way to manufacture tablets and granules. The great advantages of this method are the low manufacturing cost, conventional equipment, commonly available excipients and a limited number of processing steps involved in direct compression. 
- Dry Granulation (Slugging) method: Dry granulation also referred to as precompression or double compression is a size enlargement process designed to improve the flow and compression characteristics of powders that would otherwise be unsuitable for compression. The process involves compaction of powder particles into large pieces or compacts which are subsequently broken down into granules to produce granules that can be further processed into dosage forms.

- Wet Granulation (wet massing) method: is a process of size enlargement in which fine powder particles are agglomerated or brought together into larger, strong and relatively permanent structure called granules using a suitable non-toxic granulating fluid such as water or ethanol. Powder mixing, in conjunction with the cohesive properties of the granulating agent, enables the formation of granules. The characteristics and performance of the final product, greatly depends on the extent to which the powder particles interact with each other to form aggregates (granules). Therefore wet granulation technique uses the same preparatory and finishing steps of direct compression and dry granulation (dry screening and mixing); with additional steps of wet massing, wet screening and drying.

\section{Methods}

\subsection{Materials}

Valsartan was obtained as a gift powder from Awamedica Pharmaceutical Company, Erbil/ Iraq, polyvinyl pyrollidone, lactose, orange flavour, sorbitol and distilled water were also used.

\subsection{Instruments used in the study}

The instruments used in the study include sensitive balance, melting point apparatus, $\mathrm{pH}$ meter, UV-spectrophotometer, FTIR, distillator, dissolution apparatus type II paddle, magnetic stirrer and shaker.

\subsection{Melting point determination}

The melting point of valsartan was measured by using capillary tube method.
A tiny amount of valsartan powder was introduced in to a small glass capillary tube $10-15 \mathrm{~cm}$ long, which is closed at one end, attaching this to the stem of a thermometer centred in a melting point apparatus. The sample was heated slowly, while the sample was carefully observed. The temperature at which melting begins and complete was recorded as a melting point. ${ }^{12}$

\subsection{Determination of calibration curve} of valsartan

2.4.1.Preparation of standard stock solution $^{13}$

Standard drug solution of valsartan was prepared by dissolving $10 \mathrm{mg}$ pure valsartan in methanol and distilled water then transferred into $100 \mathrm{ml}$ volumetric flask to obtain $100 \mu \mathrm{g} / \mathrm{ml}$ of stock solution from which desired concentrations of solutions were prepared.

\subsubsection{Determination of calibration curve of valsartan ${ }^{14}$}

Standard stock solution was suitably diluted with distilled water to obtain concentrations ranging from $1-10 \mu \mathrm{g} / \mathrm{ml}$. Absorbance of these solutions was measured at $255 \mathrm{~nm}$ ( $\lambda$ max of valsartan) using UV-spectrophotometer, calibration curve was obtained by plotting graph between concentration and absorbance.

\subsection{Drug-excipient compatibility study Fourier Transforms Infrared Spectroscopy (FT-IR) Studies:}

The FT-IR (Jasco, FT/ IR- 4600)) was used for the IR analyses in the frequency range between 4000 and $400 \mathrm{~cm}-1$ and at $1 \mathrm{~cm}^{-1}$ resolution. The instrument was calibrated by using polystyrene film. Pure drug and excipients were selected separately. ${ }^{15}$ The IR peaks of pure valsartan were analyzed and compared with the peaks of the obtained formulations (F1 and F2).

2.6. Formulation of valsartan sachet using wet granulation method:

Wet granulation method was used to prepare two different formulations of valsartan sachet named novel formula (F1) and conventional formula (F2) as shown in Table1. Both formulas were prepared by 
the same ingredients and method, the only differences between them was in the technique of mixing. For F1 which is the novel formula prepared by dissolving the drug and polyvinyl pyrollidone (PVP) separately in small quantities of ethanol and distilled water (D.W), respectively, then these liquid mixtures were mixed together and finally added to the previously thoroughly mixed dry ingredients. While for F2 which is the conventional formula, only the PVP was dissolved in small quantity of distilled water then the formed liquid mixture was added to the remaining previously mixed dry ingredients. After preparation of both formulas, all ingredients were passed through mesh \# 60 and dried in the oven for 30 minutes to get a granules and form a sachet.

\subsection{Post preparation evaluation parameters of valsartan sachet}

2.7.1. Weight variation test:

Ten sachets were taken and their weight was determined individually and collectively on a digital weighing balance. The average weight of one sachet was determined from the collective weight. ${ }^{16}$

\subsubsection{Weight content uniformity:}

Six sachets of $300 \mathrm{mg}$ equivalent weight of valsartan dry granules was accurately weighed and transferred into a $100 \mathrm{ml}$ volumetric flask containing $(20 \mathrm{ml}$ ethanol and $80 \mathrm{ml}$ buffer solution). The solution in the volumetric flask was shaked for 10 minutes using magnetic stirrer and shaker then filtered, diluted suitably and drug content was analyzed using UVVisible spectrophotometer (analytiKjena, SPECORD 40) at $\lambda \max 255 \mathrm{~nm} .{ }^{16}$

\subsubsection{Dissolution test:}

The release of valsartan sachet was determined using USP dissolution testing apparatus type II (paddle method). The dissolution test was performed using 900 $\mathrm{ml}$ of phosphate buffer solution $(\mathrm{pH} \mathrm{6.8)}$ at $37 \pm 0.1^{\circ} \mathrm{C}$ and $50 \mathrm{rpm}$. A sample $(5 \mathrm{ml})$ of the solution was withdrawn from the dissolution apparatus at different time intervals and the samples were replaced with fresh dissolution medium. The samples were filtered through a $0.45 \mu$ membrane filter and diluted to suitable concentration with buffer solution ( $\mathrm{pH} 6.8)$. Absorbance of these solutions was measured at $\lambda \max 255 \mathrm{~nm}$ using a UVvisible spectrophotometer. Cumulative percentage drug release was calculated using regression equation obtained from a standard curve. ${ }^{16}$

\subsubsection{Comparison with marketed} product (diovan):

The release characteristics of valsartan sachet (F1 and F2) were compared with available marketing product of valsartan tablet (diovan) ${ }^{R}$

\subsection{Statistical Analysis}

All experiments were carried out in triplicate. The values were represented as mean \pm standard deviation. The differences in release profile of new developed formula (F1) and marketed conventional tablet were tested for significance by using independent two samples t-test with the aid of the statistical package for the social sciences (version 18) program. Differences were considered statistically significant when $(P<0.05)$.

Table 1: Formulation of valsartan oral granule (sachet).

\begin{tabular}{lcccccc}
\hline Formula name & Valsartan & PVP & Orange flavour & Sorbitol & Lactose & Total (mg) \\
\hline F1 & 40 & 2.5 & 5 & 20 & 232.5 & 300 \\
F2 & 40 & 2.5 & 5 & 20 & 232.5 & 300 \\
\hline
\end{tabular}




\section{Results}

\subsection{Melting point determination}

The measured melting point of valsartan was $115-120^{\circ} \mathrm{C}$ which is within the reported value of $116^{\circ} \mathrm{C} ;{ }^{17}$ this indicates the rational purity of drug powder.

3.2. Determination of $\lambda$ max (maximum absorbance)

Valsqartan solution was scanned by UV-spectrophotometer at a wave length of 200-400 nm in $1 \mathrm{~cm}$ cell. The spectrum reveals that valsartan shows a well defined $\lambda_{\max }$ at $255 \mathrm{~nm},{ }^{18}$ as shown in Figure 1.

\subsection{Determination of calibration curve of} valsartan

Calibration curve data and calibration curve parameters for valsartan in methanol with distilled water analyzed by UVspectrophotometer at $255 \mathrm{~nm}$ demonstrate that calibration curve was linear in the concentration range from 1 to $10 \mu \mathrm{g} / \mathrm{ml}$. The correlation coefficient was found to be (0.999) and the calibration plot is illustrated in Figure 2.

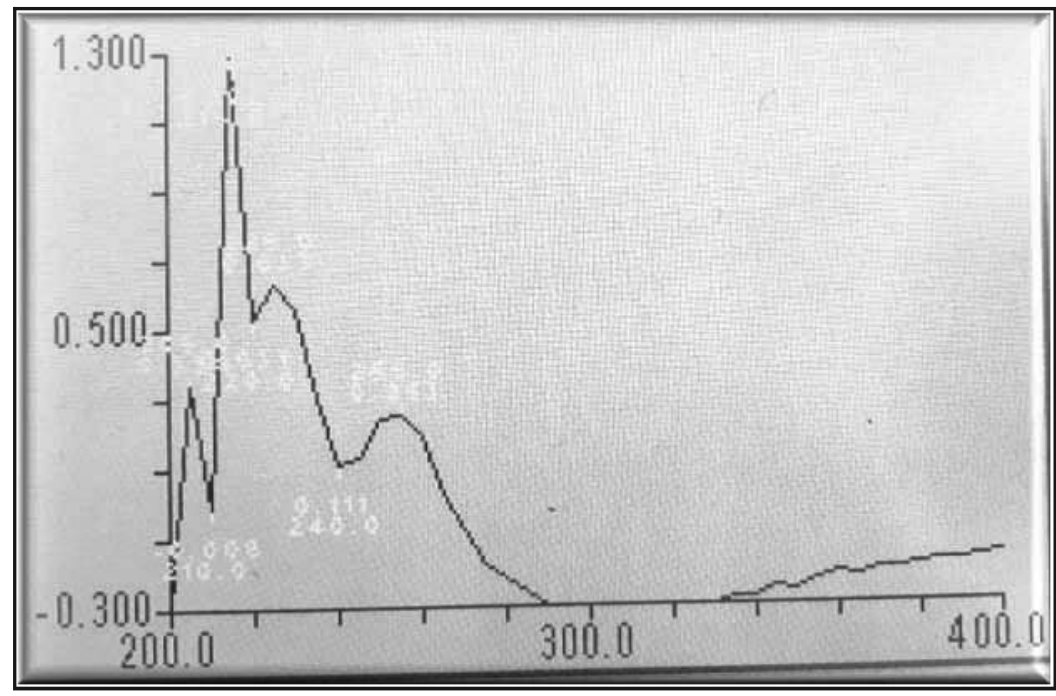

Figure 1: UV-Scan of valsartan in methanol with phosphate buffer ( $\mathrm{pH} 6.8)$.

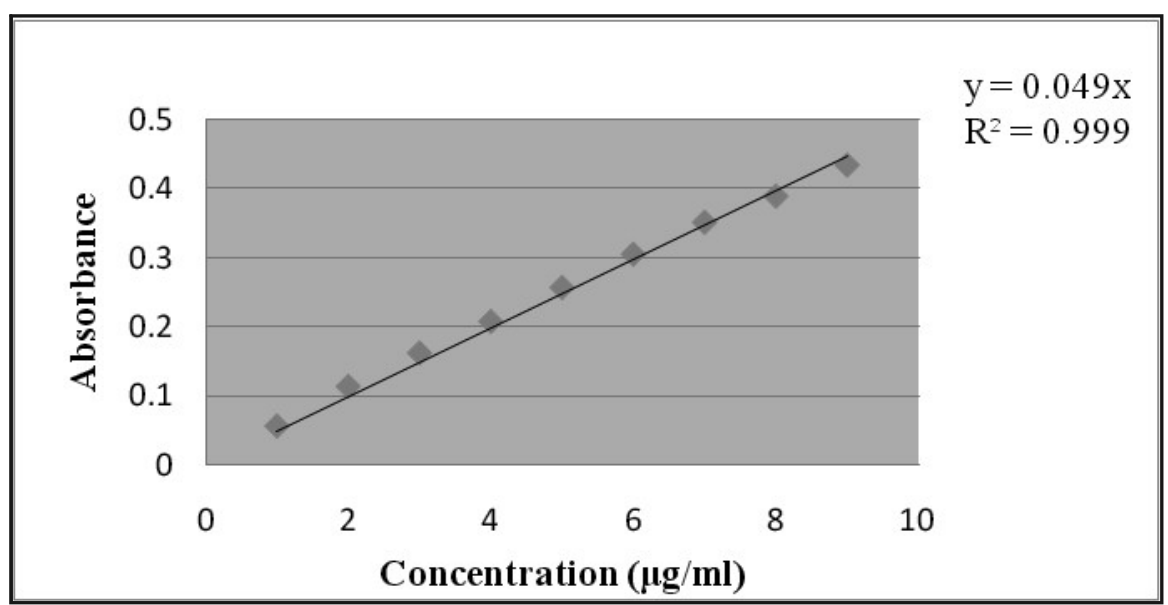

Figure 2: Calibration curve of valsartan in methanol with phosphate buffer $(\mathrm{pH} \mathrm{6.8)}$. 
3.4.Drug-excipient compatibility study Fourier Transformation Infra-red (FTIR) analysis

This study was done by FTIR to evaluate the compatibility of valsartan with pharmaceutical excipients of common use as diluents and binder. The infrared (IR) spectra of pure valsartan drug powder and the prepared formulas (F1 and F2) were taken for the characterization studies as shown in figures (3), (4) and (5), respectively. IR spectrum of pure valsartan powder showed characteristic peaks at $2963.09 \mathrm{~cm}^{-1}$ due to $\mathrm{C}=\mathrm{N}$ stretching, at $1731.76 \mathrm{~cm}^{-1}$ due to carboxylate stretching. The spectra also showed bands at 1731.76 $\mathrm{cm}^{-1}$ due to $\mathrm{C}=\mathrm{O}$ bending, at $1105.01 \mathrm{~cm}^{-1}$ due to $\mathrm{C}-\mathrm{N}$ bonding. ${ }^{19}$

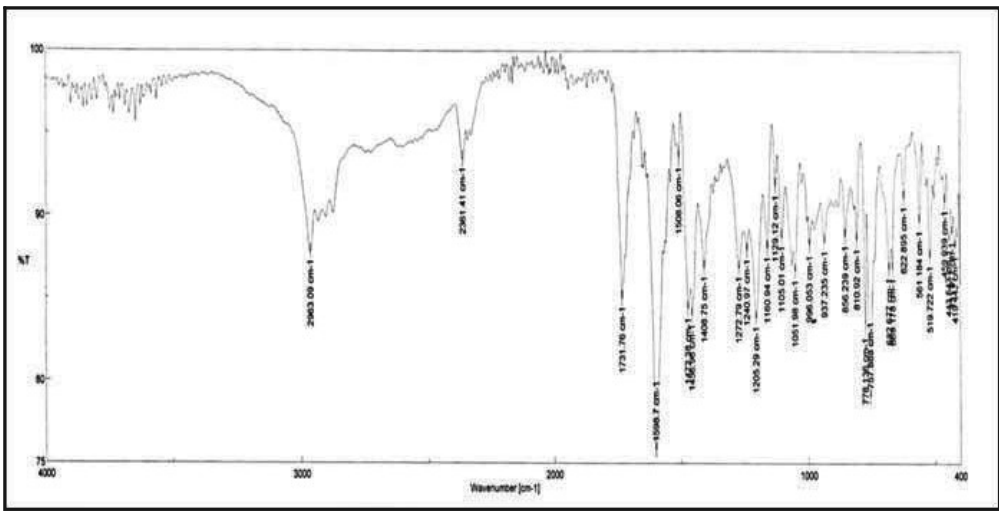

Figure 3: FTIR spectrum of pure valsartan powder.

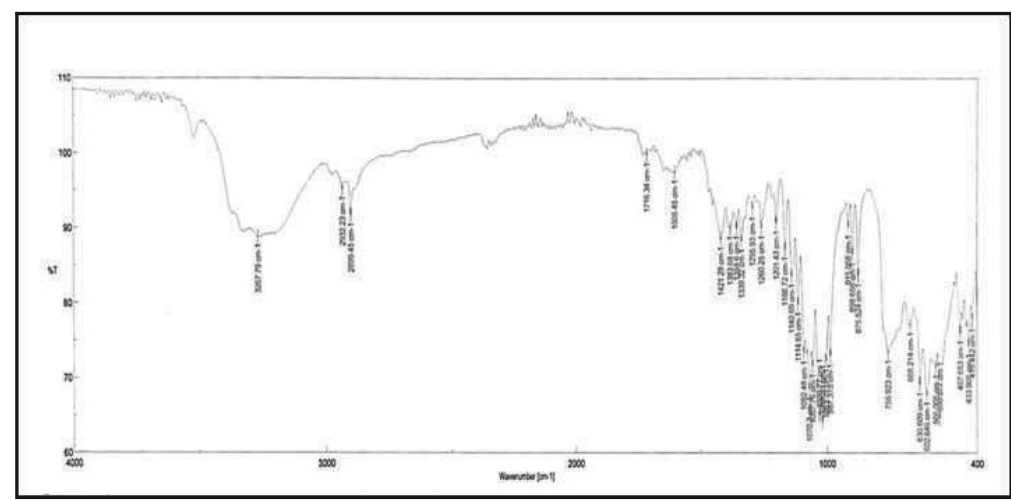

Figure 4: FTIR spectrum of F1.

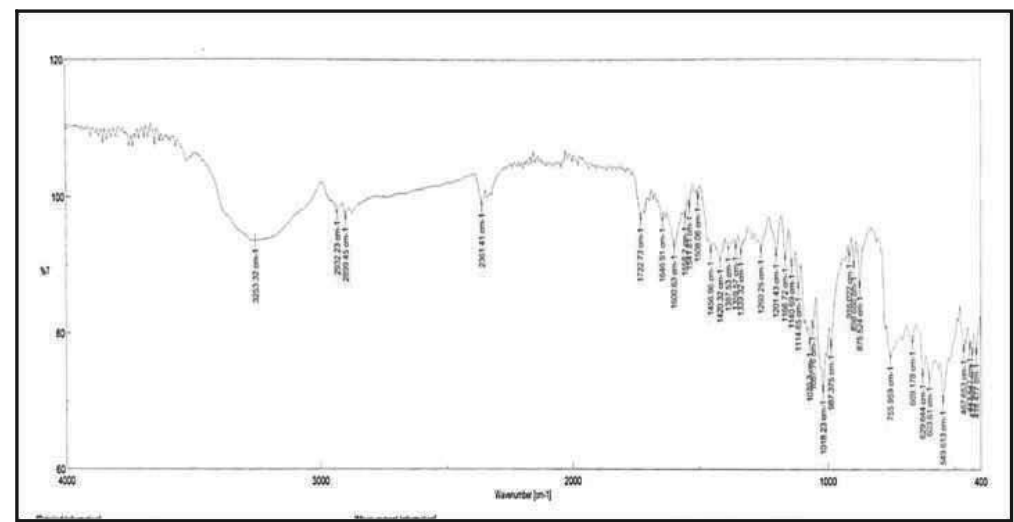

Figure 5: FTIR spectrum of F2. 
3.5. Post preparation evaluation parameters of valsartan sachet

3.5.1.Weightvariatioen test:

Weight variation of the prepared valsartan sachet was determined and listed in Table 2. The result show that there was a non-significant differences $(P>0.05)$ between $\mathrm{F} 1$ and $\mathrm{F} 2$ and the results are expressed as a mean \pm S.D $(n=10)$.

\subsubsection{Weight content uniformity:}

The calculated percentage of the weight content uniformity of the prepared formulas $\mathrm{F} 1$ and F2 with diovan are listed in Table 3. Where there was a non-significant differences $(P>0.05)$ between the prepared valsartan sachet and diovan, also the results are expressed as a mean \pm S.D $(n=6)$.

3.5.3.Dissolution test of $F 1$ and $F 2$ in comparison with diovan:

The dissolution test of the novel (F1) and conventional (F2) formulas of the prepared valsartan sachet as well as valsartan conventional tablet (Diovan ${ }^{\circledR} 80 \mathrm{mg}$ tablet) as a reference was done according to USP, using $900 \mathrm{ml}$ of phosphate buffer solution $(\mathrm{pH} 6.8)$ as a dissolution medium at $37^{\circ} \mathrm{C}$ with constant stirring speed of 50 rpm for 45 minutes and the results listed in Table 4 and are expressed as a mean \pm S.D $(n=6)$.

Table 2: Weight variation test of F1 and F2 with diovan.

Formula name Weight variation (mg)

$\mathrm{F} 1$

F2

Diovan
$299.4+4.86$

$296.9 \pm 4.39$

$80.9 \pm 6.54$

Table 3: Weight content uniformity of $\mathrm{F} 1$ and $\mathrm{F} 2$ formulations with diovan.

Formula name

$96.06 \pm 1.96$

$95.93 \pm 3.31$

Weight uniformity (mg)
F2

F1

2

Diovan
$95.45 \pm 1.43$ 
Figure 6 revealed the comparison of the release profile of the prepared valsartan sachet (F1 and F2) with that of the valsartan conventional tablet using diovanas a reference. Depending on the USP pharmacopeia for the solid dosage forms 30 minutes is needed to release
$75 \%$ of the drug content. ${ }^{12}$ The results indicate that within 30 minutes about $96.84 \%$ and $78.24 \%$ of drug was released from $\mathrm{F} 1$ and diovan respectively which is within the acceptable limit, while for F2 only $64.60 \%$ of the drug was released within the same time.

Table 4: Dissolution profile of F1 and F2 with diovan.

\begin{tabular}{cccc}
\hline Time (min.) & $\begin{array}{c}\text { Percentage of release } \\
\text { of F1 (\%) }\end{array}$ & $\begin{array}{c}\text { Percentage of release } \\
\text { of F2 (\%) }\end{array}$ & $\begin{array}{c}\text { Percentage of release } \\
\text { of diovan (\%) }\end{array}$ \\
\hline 10 & 55.51 & 35.7 & 40.19 \\
20 & 77.18 & 50.77 & 60.97 \\
30 & 96.84 & 64.6 & 78.24 \\
45 & 86.09 & 37.55 & 68.95 \\
\hline
\end{tabular}

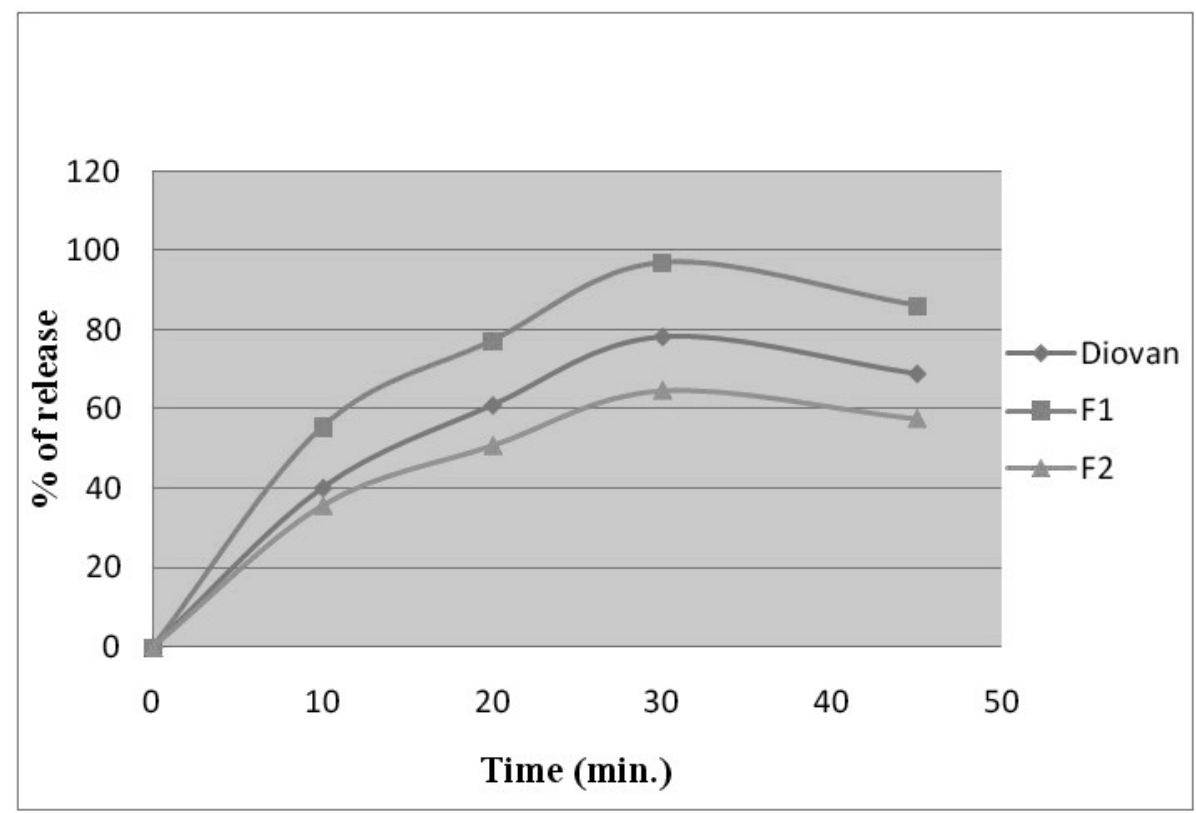

Figure 6: Dissolution profile of F1 and F2 with diovan. 


\section{References}

Pre-preparation evaluation parameters of pure valsartan powder:

The rational purity of pure valsartan powder indicated by the result obtained from the measurement of melting point with the measurement of the maximum absorbance and the preparation of the calibration curve.

Post preparation evaluation parameters of valsartan sachet:

Post preparation evaluation parameters were done for the prepared formulas F1 and F2 with the conventional tablet of valsartan using diovan as a reference.

Drug-excipient compatibility study:

Fourier Transformation Infra-red (FTIR) analysis

The comparison of the IR spectrum of F1 and F2 with that of pure drug showed all the characteristic peaks of pure valsartan and revealed that there is no appreciable change in the positions of characteristic absorption bands of groups and bonds. The spectra of these, even though slightly differ in appearance but no change is observed in the positions of the bands in the spectra. This clearly suggests that the drug remains in the same form even in its formulations indicating that there is no interaction between the drug and excipients used for the preparation of valsartan sachet.

\section{Weight variatioen test:}

The results of weight variation test indicated that both formulas (F1 and F2) were within pharmacopoeial limits (not more than two tablets differ from the average by no more than $10 \%$ and no tablets differ by more than $20 \%),{ }^{19}$ the result indicate that there was a nonsignificant differences $(P>0.05)$ between $\mathrm{F} 1$ and $\mathrm{F} 2$.

\section{Weight content uniformity:}

The drug content uniformity of the prepared valsartan sachet (F1 and F2) complies with USP criteria; it was found that no sachet from ten sachets lies out of the label claim, ${ }^{19}$ which indicates a uniform distribution and a proper dose of the drug in the sachet. Therefore the results indicate that there was a non-significant differences $(P>0.05)$ between $\mathrm{F} 1$ and $\mathrm{F} 2$ with diovan.

Dissolution test of $F 1$ and $F 2$ in comparison with diovan:

The rate and extent of dissolution of the active ingredient from any dosage form often determines the rate and extent of absorption of the drug. $^{20}$ In case of valsartan which has low water solubility, dissolution may be the rate- limiting step in the process of drug absorption; therefore drugs with low water solubility have been shown to be unpredictably and slowly absorbed compared with drugs of higher solubility and a better oral formulation like valsartan sachet (F1 and F2) can be developed by increasing the water solubility of such drugs. Based on the USP pharmacopeia for the solid dosage forms 30 minutes is needed to release $75 \%$ of the drug content. ${ }^{12}$ The results indicate that within 30 minutes about $96.84 \%$ and $78.24 \%$ of drug was released from $\mathrm{F} 1$ and diovan respectively which is within the acceptable limit, while for F2 only $64.60 \%$ of the drug is released within the same time. Also the results showed that after 10 , 20 , and 45 minutes the percentage of drug release from $\mathrm{F} 1$ was $55.51 \%, 77.18 \%$ and $86.09 \%$ respectively and for diovan was $40.19 \%, 60.97 \%$, and $68.96 \%$ respectively. Whereas the percentage of drug release from $\mathrm{F} 2$ for the same time was $35.70 \%, 50.77 \%$ and $57.85 \%$ respectively. So, this indicates that there was a significant differences $(P<0.05)$ in the dissolution of the novel formula (F1) which was faster than the conventional formula (F2) of the prepared valsartan sachet, while a non- significant differences $(P>0.05)$ was obtained between $\mathrm{F} 1$ and the conventional valsartan reference tablets (Diovan $^{\circledR} 80 \mathrm{mg}$ tablet). This significant improvement in the dissolution of the prepared valsartan sachet can be related to that F1 does not need disintegration process because is a granule dosage form and it is known that decreasing of the disintegration time leads to increase in dissolution rate ${ }^{21}$ because 
faster disintegration delivers a fine suspension of drug particles resulting in a higher surface area and faster dissolution. ${ }^{22}$ While the same improvement not observed with $\mathrm{F} 2$ due to the problem of solubility of valsartan which is within the class II drugs depend in on biopharmaceutical classification system (BCS). Therefore F2 needs improvement in its solubility before preparation as a sachet or any other dosage form.

\section{Conclusion}

\section{Conclusion}

Valsartan oral granule as sachet or dry granules can be used as alternative dosage form The prepared novel formula F1 provided a significant improvement in the drug release profile in comparison with conventional formula F2 and the marketed conventional valsartan tablet. The overall results indicates the possibility of using the novel formula F1 in the preparation of valsartan oral granules, as a new dosage form for the oral administration with fast dissolution time, that can occupy the place of the conventional valsartan tablets.

\section{Competing interests}

The authors declare that they have no competing interests.

\section{References}

1. Tripathi KD. Essentials of Medical Pharmacology 2005; 5 th Edition: 476-554.

2. Alfred Goodman Gilman, Theodore W. Rall, Alan S. Nies, Palmer Taylor. The Pharmacological Basis of Therapeutics, Eighth Edition, Volume II 50-64.

3. Flesch G, Muller P, Lloyd P. Absolute bioavailability and pharmacokinetics of valsartan, an angiotensin II receptor antagonist, in man. Eur J Clin Pharmacol 1997; 52(2):115-120.

4. Mali RR, Goel V, Gupta S. Novel study in sustained release drug delivery system: A Review. Int J Pharm Med Res 2015; 3(2):20415.

5. Kundu S, Patil AV, Srinivasan G, Borkar N. Controlled Release Suspension: A Review. International Journal of Pharmaceutical Innovations 2011; 2(4):1-18.

6. Patel M, Patel KR, Patel MR, Patel NM. Formulation and Evaluation of Microemulsion Based Gel of Ketoconazole International Journal of Universal Pharmacy and Bio Sciences 2014; 3(2):93-111.

7. Bardeskar C, Geeverghese R. Reconstitutable Oral Suspensions (Dry Syrups): An Overview. World Journal of Pharmaceutical Research. 2014; 4(3):462-84.

8. Damor SR, Jethara SI, Patel MS, Patel MR. A Review On Dual Release Oral Reconstitutable Suspension. World Journal of Pharmaceutical Research. 2015; 4(3):592-613.

9. Bhandare P, Yadav A. A Review on "Dry Syrups ForPaediatrics". Int J Curr Pharm Res 2015; 9(1):25-31.

10. Pavane $M$, Shirsat $M$, Dhobale $A$, Joshi $D$, Dhembre G, Ingale P. Formulation, Development and Evaluation of Oral Reconstitutable Dry Syrup. Indo American Journal of Pharmaceutical Sciences 2018; 5(1):483-91.

11. Tsue $S$, Abe S, Shimotori $T$, Sugisawa $K$. Evaluation of Direct Compression Tablet prepared by a new Super fine Powder of (HPC) Hydroxypropyl Cellulose. Nihongi Laboretory, Nippon Soda Co., Ltd., Japan 2011.

12. USP-30 NF-25 (2007), United State Pharmacopoeial Convention. Electronic Version.

13. Kishanta Kumar Pradhan and U. S. Mishra. Development and validation of stability indicating RP-HPLC method for the determination of valsartan. Int. J. of Pharmacy and pharmaceutical sciences 2015; 7(8): 57-61.

14. Nataraj KS, Ramakrishnama Charya SV, Swathi Goud E, Saigeethika S, Ramanjineyulu K. Simple quantitative method development and validation of valsartan in pureform and pharmaceutical dosage forms byuv spectroscopy. International Journal of Pharmacy and Biological Sciences. IJPBS 2011; 1(2).

15. Usha Sri B, Indira Muzib Y, DVRIN Bhikshapathi, Sravani R. Enhancement of solubility and oral bioavailability of poorly soluble drug valsartan by novel solid self emulsifying drug delivery system. Int $\mathrm{J}$ of drug delivery 2015; 7:13-26.

16. Bhabani Shankar Nayak, Sruti Ranjan Mishra, Harekrishna Roy. Valsartan Fast Dissolving Tablets: Formulation and In vitro Characterization. Journal of Chemical and Pharmaceutical Research 2018; 10(3):182-9.

17. Clarke's Analysis of Drugs and Poisons (2011). Electrical edition, pharmaceutcal press.

18. Khan F, Lohiya RT, Umekar MJ. Development of UV Spectrophotometric method for the simultaneous estimation of Meloxicam and Paracetamol in tablet by simultaneous Equation, Absorbance ratio and Absorbance Correction method. Int J Chem Tech Res 2010; 2(3):158691.

19. Balamuralidhara V, Sreenivas SA, Gangadhara $\mathrm{HV}$, Pramodkumar TM. Investigation on the Effect of Different Disintegrants on the Orodispersible Tablets of rabeprazole. Asian $\mathrm{J}$ Sci Res 2009; 4:12-9. 
20. El-badry M, Fathy M. Enhancement of dissolution and permeation rates of meloxicam by formation of its freeze dried solid dispersion in polyvinyl pyrrolidine K-30. Drug Devlnd Pharm 2006. P. 141-2.

21. Takeuchi $H$, Tanimura $S$, Nagira $S$, Yamamoto $H$ Kawashina Y. Tableting of Solid Dispersion Particles Consisiting of Indomathacin and Porous Silica Particles. Chem Pharm Bull 2005; 53 (5):487-91.

22. Malikarjuna SC, Prasad DV, Gupta VR. Development of fast dispersible aceclofenac tablets: Effect of functionality of super disintegrants. Ind J pharm Sci 2008; 70(2):180-5. 